\title{
Synthesis of Frequency-Independent Phase Shifters Using Negative Group Delay Active circuit
}

\author{
Blaise Ravelo ${ }^{1}$, André Pérennec ${ }^{2}$, Marc Le Roy $^{2}$ \\ ${ }^{1}$ IRSEEM, ESIGELEC, Technopole du Madrillet, Avenue Galilée - BP10024. 76801 Saint \\ Etienne du Rouvray Cedex, France. \\ ${ }^{2}$ Université Européenne de Bretagne (UEB), Université de Bretagne Occidentale (UBO) \\ Lab-STICC UMR CNRS 3192, 6 Av. Le Gorgeu, CS93837, 29238 Brest cedex 3, France.
}

\begin{abstract}
This paper describes a synthesis method dedicated to the design of frequency-independent phase shifters (PS). This innovative PS structure consists in a transmission line cascaded with a negative group delay (NGD) active circuit so that the absolute constant group delays generated by both of them are identical, but of opposite signs. So, in principle, it exhibits a constant overall phase and a group delay close to zero. Broadband linear positive phase slopes are obtained through use of an NGD active circuit whose characteristics are recalled prior to the extraction of the PS synthesis relations. The design and simulations of a PS of compact size are reported. The experimental results confirm the expected frequency-independent transmission-phase value of $145^{\circ} \pm 10^{\circ}$ with an insertion gain of $2 \pm 2 \mathrm{~dB}$ over a $160 \%$ relative frequency band. At last, future prospects allowed by the specific properties of this PS are presented.
\end{abstract}

Keywords: Active circuit, broadband, constant phase, negative group delay (NGD), phase shifter (PS), synthesis method.

\section{INTRODUCTION}

Since the 60's, several theoretical and experimental studies [1-13] have demonstrated the existence of negative group velocity, and thus of negative group delay (NGD). Among them, it is worth citing the investigations carried out in optical- [1-5], electronic- [6-8] and even acoustic-domains [9-10]. These experiments evidenced that the occurrence of this counterintuitive phenomenon does not contradict the causality principle. In 2003, this phenomenon was also confirmed in the microwave area with passive circuits [11-13]. But, in 
this domain, the existing NGD passive circuits are not suitable for microwave applications because excessive losses inherent to the NGD phenomenon are present.

To overcome this issue, a topology of microwave active circuit able to provide simultaneously a significant NGD and gain was recently introduced and experimentally validated [14-15]. It simply consists of a field effect transistor (FET) in cascade with a shunt series RLC passive network as depicted in Figure 1.

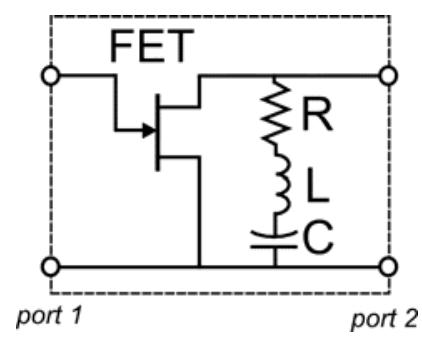

Figure 1. NGD cell: FET cascaded by shunt RLC resonant network.

As shown theoretically in [14], this circuit provides a group delay, which is always negative at the resonance angular frequency, $\omega_{0}=1 / \sqrt{L C}$. Then, in [15], a broadband NGD circuit with loss compensation was designed through the use of a specified synthesis method and implemented; this first verification in microwave domain proved to be promising. Indeed, the investigations briefly introduced in [16-17] were about the application of such NGD cells in the design of phase shifter (PS) that exhibits a frequency-independent transmission-phase value, $\varphi(\omega)=C^{t e}$. Then, the corresponding group delay, $\tau(\omega)=-\partial \varphi(\omega) / \partial \omega$ is mathematically equal to zero. This new structure of PS consists in cascading a classical transmission line with a NGD active circuit whose absolute phase slopes are identical in absolute value, but their signs in the operating frequency band are opposite (see Figure 2). Thus, in this band, the resulting phase is constant. In other words, the transmission line (TL) has a positive constant group delay (PGD) and the active circuit must provide an opposite constant negative group delay value as depicted in Figure 2(b). 


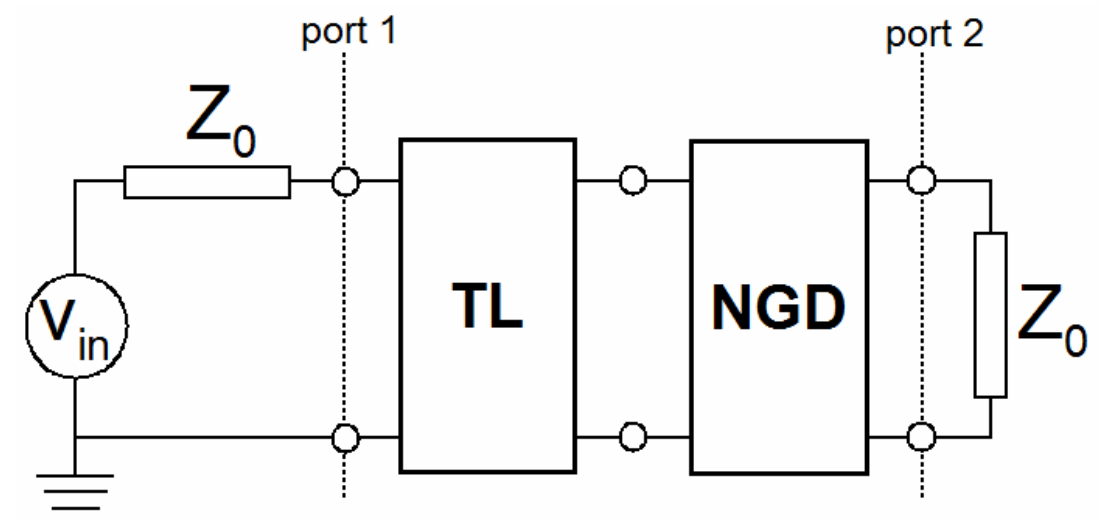

(a)

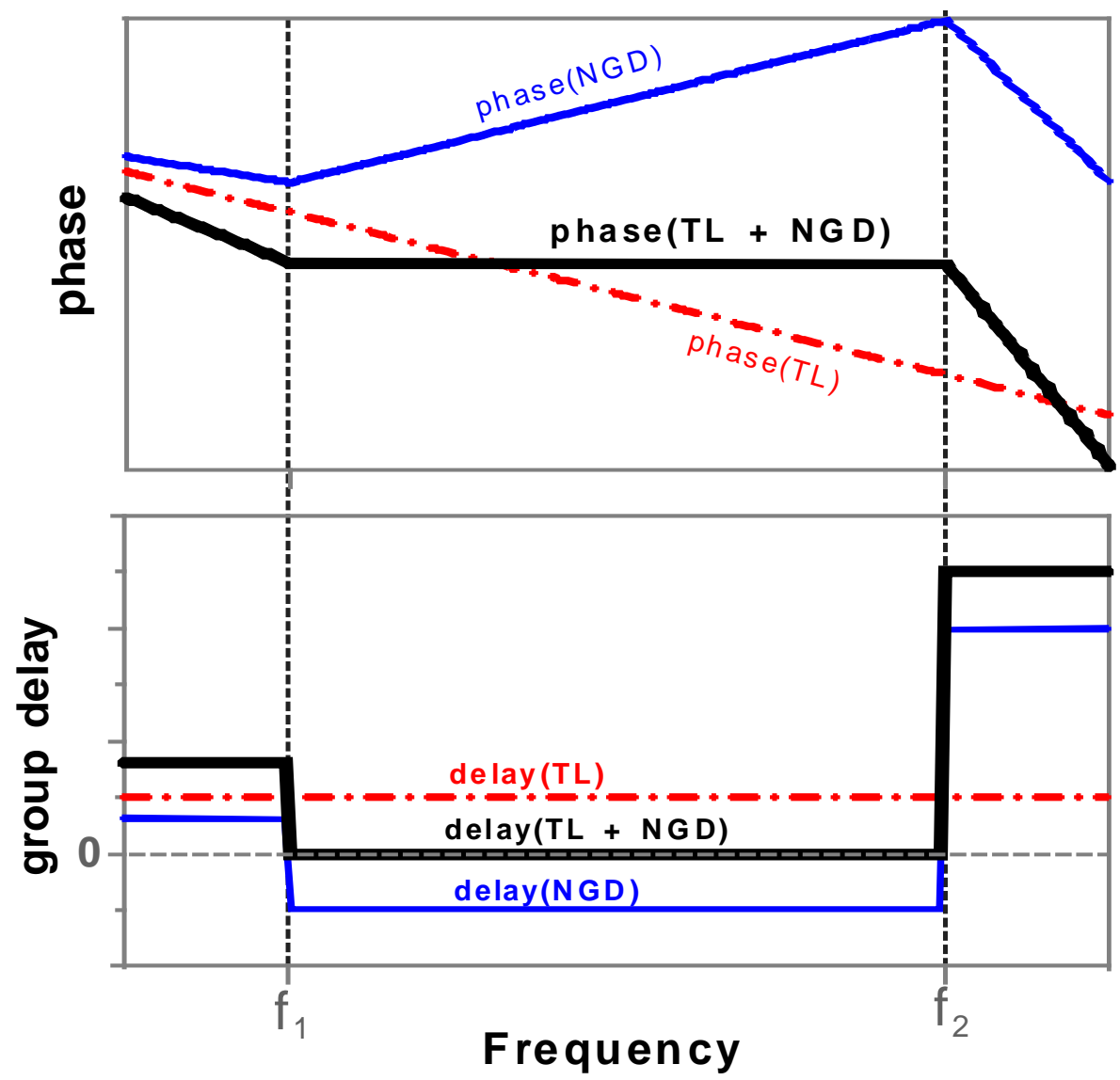

(b)

Figure 2(a). Structure of the proposed NGD PS: transmission line (TL) cascaded with the NGD circuit and (b) ideal behavior of their phase and group delay responses.

Historically, the basic concept of constant or frequency-independent PS was proposed at first, by Hartley [18] for the audio frequency range to be mainly used in SSB modulation 
systems. Later, Kiyasu [19] and Darlington [20] both published a theoretical concept of constant PS. Then, in $1977,90^{\circ}$-PS based on distributed- and lumped-elements were implemented for VHF band. In addition, over the period 1970-1982 several papers and patents presented by Phelan [21-22] described a frequency-independent PS through use of a reflect array approach, called the "spiraphase". But, this approach is useful only in relative band narrower than $10 \%$ [23]. So, to overcome this limitation, phase shifters were proposed by Wang in [24-25]: their specificities are the use of PV-FET (photovoltaic FET) switches controlled by optical fibers and the production of frequency-independent performances, but their implementation in hybrid technology is complex. It is worth recalling that a broadband PS is among the key components within phased-array antennas, radar and satellite broadcasting/communication systems. This consideration has led to the fabrication of a constant PS based on RF MEMS for X-Band [26], but losses cannot be neglected and its bandwidth is somewhat limited.

In addition to being able to compensate for losses, the constant PS proposed in this paper is easy to implement and integrate, attractive in terms of compactness and potentially wideband. It relies on the simple principle schematized in Figure 2. The underlying concept of constant PS with NGD circuit was briefly introduced in [16]. On the other hand, the present paper is aimed at fully describing the concept, the design as well as the synthesis method while showing how to determine the PS parameters as a function of the phase, bandwidth, and gain values. Moreover, it also deals with the design, from these synthesis relations, and the fabrication of a proof-of-concept broadband PS with an operating frequency band wider than that of the PS proposed in [16]. The behavior of the NGD PS is confirmed by the good agreement found between simulation results and measurements. One should also note that, in addition to the benefits mentioned above, this PS exhibits a singular property: a constant transmission phase (group delay close to zero) is generated instead of a constant phase difference as encountered in certain classical PSs [26-28]. 
This paper is organized as follows: the expressions of NGD cell S-parameters are recalled in section 2 prior to the calculation of the PS ones and to the establishment of the synthesis relations according to the given specific characteristics. Section 3 reports on the design and fabrication of the proof-of-concept PS from the established synthesis relations. Further to the comparison of simulation results and measurements also in section 3 , conclusions are drawn in section 4 together with suggestions about potential applications.

\section{THEORY}

Figure 3 shows the configuration of the constant PS under study. As previously mentioned, an NGD circuit resonating at $\omega_{0}=1 / \sqrt{L C}$ is cascaded at the output of a transmission line (TL), which plays the role of the PGD element.

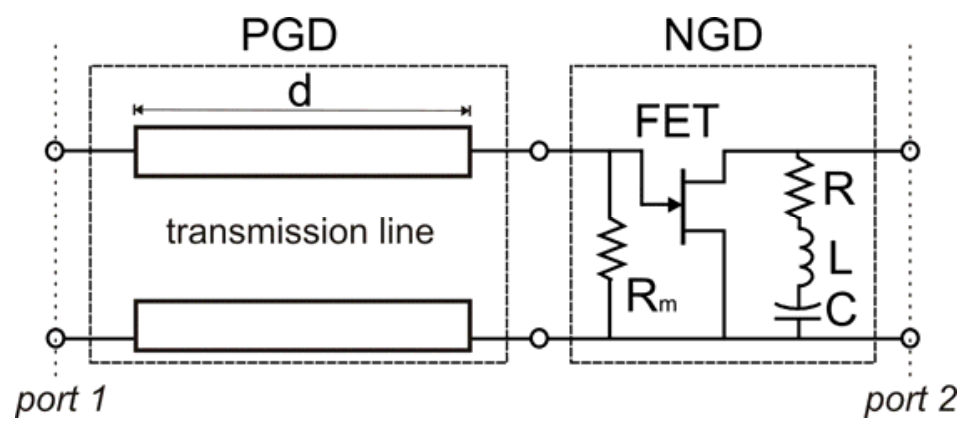

Figure 3. Structure of the PS under study: transmission line cascaded by an NGD cell.

To simplify this theoretical study, let us assume that, at the angular frequency, $\omega_{1}=2 \pi f_{1}$, the quarter-wavelength line of length $d$ is lossless and printed on a substrate of effective permittivity, $\varepsilon_{\text {eff }}$. Moreover, its characteristic impedance is: $Z_{\theta}=50 \Omega$. The transmission parameter is then expressed as: 


$$
S_{21 T L}(j \omega)=e^{-j \pi \frac{\omega}{2 \omega_{1}}}
$$

with

$$
\omega_{1}=\frac{\pi c}{2 d \sqrt{\varepsilon_{e f f}}}
$$

where $c$ is the speed of light in vacuum.

To improve the inter-stage matching between the TL and the NGD cell of the structure displayed in Figure 3, a shunt resistance $R_{m}$ is connected to the input of the latter. For simplification of the calculation let us consider the low-frequency model of the FET with a voltage-controlled current source of transconductance, $g_{m}$, and the drain-source resistor, $R_{d s}$, depicted in Figure 4 with $R_{m}$ and the RLC network.

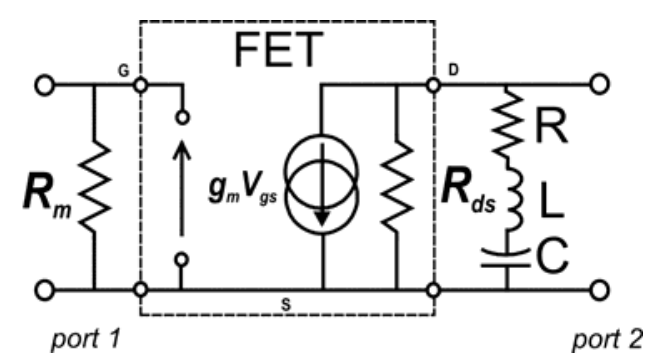

Figure 4. NGD cell using the FET low-frequency model.

The theoretical expressions of S-parameters for this circuit were previously determined and are available for this configuration in [15].

\subsection{NGD PS S-Parameters}

Figure 3 shows that the ABCD chain matrix of the PS results from the multiplication of TL one by that of the NGD circuit. Thus, by using the conversion relationships between the ABCD-matrix and the S-parameters, one gets the PS S-parameters

$$
S_{11}(j \omega)=e^{-j \pi \frac{\omega}{\omega_{1}}} S_{11 N G D}(j \omega),
$$




$$
\begin{aligned}
& S_{21}(j \omega)=e^{-j \pi \frac{\omega}{2 \omega_{1}}} S_{21 N G D}(j \omega)=S_{21 T L}(j \omega) . S_{21 N G D}(j \omega), \\
& S_{22}(j \omega)=S_{22 N G D}(j \omega) .
\end{aligned}
$$

One should note that these equations are still valid in the case of imperfectly matched stages.

Obviously, eq. (4) allows one to get the gain and the transmission phase denoted as follows:

$$
\begin{aligned}
& \left|S_{21}(j \omega)\right|=\left|S_{21 N G D}(j \omega)\right|, \\
& \varphi(\omega)=\varphi_{T L}(\omega)+\varphi_{N G D}(\omega),
\end{aligned}
$$

where $\varphi_{T L}$ and $\varphi_{N G D}$ are, respectively, the TL and NGD-circuit transmission phases resulting from eq. (1) and from [15]:

$$
\begin{aligned}
& \varphi_{T L}(\omega)=-\pi \frac{\omega}{2 \omega_{1}}, \\
& \varphi_{N G D}(\omega)=\pi+\operatorname{atg}\left[\frac{L\left(\omega^{2}-\omega_{0}^{2}\right)}{R \omega}\right]-\operatorname{atg}\left[\frac{L\left(Z_{0}+R_{d s}\right)\left(\omega^{2}-\omega_{0}^{2}\right)}{\omega\left[Z_{0} R_{d s}+R\left(Z_{0}+R_{d s}\right)\right.}\right],
\end{aligned}
$$

Thus, the TL group delay is expressed as:

$$
\tau_{T L}(\omega)=-\frac{\partial \varphi_{T L}(\omega)}{\partial \omega}=\frac{\pi}{2 \omega_{1}}
$$

As expected and required by the PS principle, at the resonance, $\omega=\omega_{0}$, the group delay of the NGD circuit is always negative: 


$$
\tau_{N G D}\left(\omega_{0}\right)=-\left.\frac{\partial \varphi_{N G D}(\omega)}{\partial \omega}\right|_{\omega=\omega_{0}}=\frac{-2 L Z_{0} R_{d s}}{R\left[R R_{d s}+Z_{0}\left(R+R_{d s}\right)\right]} .
$$

At the resonance frequency and for this topology, the full expressions of the NGD cell (with $R_{m}$ ) S-parameters are available in [15]. However, the synthesis of the proposed PS is detailed in the next section.

\subsection{Synthesis}

Similarly to all classical electronic functions, this synthesis depends on the specifications appropriated to the PS under design. Here, the starting point is defined by the centre angular frequency, $\omega_{0}$, the desired phase value, $\Phi$, and eventually the transmission gain, $G$. Some considerations about the choice of a FET suitable for use in an NGD cell are available in [14]. Then, to find the NGD circuit element values $(R, L$ and $C)$ for a given FET, one can invert the equation, $\left|S_{21}\left(\omega_{0}\right)\right|=G$, to get the synthesis relation for $R$ :

$$
R=\frac{G Z_{0} R_{d s}\left(Z_{0}+R_{m}\right)}{2 g_{m} Z_{0} R_{d s} R_{m}-S_{21}\left(Z_{0}+R_{d s}\right)\left(Z_{0}+R_{m}\right)}
$$

As previously described, the PS group delay must be set to zero to get a constant phase around $\omega=\omega_{0}$ :

$$
\tau_{0}=-\left.\frac{\partial \varphi(\omega)}{\partial \omega}\right|_{\omega=\omega_{0}}=\tau_{T L}\left(\omega_{0}\right)+\tau_{N G D}\left(\omega_{0}\right)
$$

Thus, from the NGD cell group delay defined in eq. (11) and on condition that $\tau_{N G D}\left(\omega_{0}\right)=-\tau_{T L}\left(\omega_{0}\right)$, eq. (13) becomes: 


$$
\tau_{0}=\frac{\pi R\left[R Z_{0}+R_{d s}\left(R+Z_{0}\right)\right]-4 Z_{0} R_{d s} L \omega_{1}}{2 R \omega_{1}\left[R Z_{0}+R_{d s}\left(R+Z_{0}\right)\right]}
$$

According to eqs. (8), (9) and (10), at the centre angular frequency, $\omega=\omega_{0}=1 / \sqrt{L C}$, the PS transmission phase, $\varphi\left(\omega_{0}\right)$, is given by:

$$
\varphi\left(\omega_{0}\right)=\pi\left(1-\frac{\omega_{0}}{2 \omega_{1}}\right)
$$

From the target phase value, $\Phi$, the TL quarter-wavelength angular frequency, $\omega_{1}$ can be calculated by inverting the equation, $\varphi\left(\omega_{0}\right)=\Phi$ :

$$
\omega_{1}=\frac{\pi \omega_{0}}{2(\pi-\Phi)}
$$

By using eqs. (2) and (16), the TL length can be expressed as:

$$
d=\frac{c(\pi-\Phi)}{\omega_{0} \sqrt{\varepsilon_{e f f}}}
$$

Furthermore, the inductance value, $L$, is obtained by inverting eq. (14) for $\tau_{0}=0$ and by using eq. (16):

$$
L=\frac{R(\pi-\Phi)\left[Z_{0}\left(R+R_{d s}\right)+R R_{d s}\right]}{2 \omega_{0} Z_{0} R_{d s}}
$$


And through the resonance expression, $\omega_{0}=1 / \sqrt{L C}$, one gets the capacitance value:

$$
C=\frac{2 Z_{0} R_{d s}}{\omega_{0} R(\pi-\Phi)\left[Z_{0}\left(R+R_{d s}\right)+R R_{d s}\right]} .
$$

Though these synthesis relations are a prerequisite for the proposed PS design, they do not guarantee a constant PS over a specified bandwidth. Let us, now, analyze the $S_{21}$ behaviour around the centre angular frequency, $\omega_{0}$.

\subsection{Linearity around the NGD PS Center Frequency}

On condition that $\Delta \omega<<\omega_{0}$, the use of the first-order Taylor series expansion around $\omega_{0}$ in the frequency band, $\left[\omega_{0}-\Delta \omega / 2, \omega_{0}+\Delta \omega / 2\right]$, leads to the following expressions for the approximated NGD gain, $\left|S_{21}(\omega)\right|$ and the phase, $\varphi(\omega)$, can be respectively expressed as:

$$
\begin{aligned}
& \left|S_{21}(\omega)\right| \approx\left|S_{21 N G D}\left(\omega_{0}\right)\right|+O\left(\omega-\omega_{0}\right)^{3}, \\
& |\varphi(\omega)| \approx \pi-\left(\omega-\omega_{0}\right) \cdot \tau_{0}+O\left(\omega-\omega_{0}\right)^{3} .
\end{aligned}
$$

From these two relations, one can deduce that the gain and the phase of the NGD cell over a limited frequency band are both nearly constant as illustrated in Figure 5. This remark applies to a cell with only a single resonant network. 


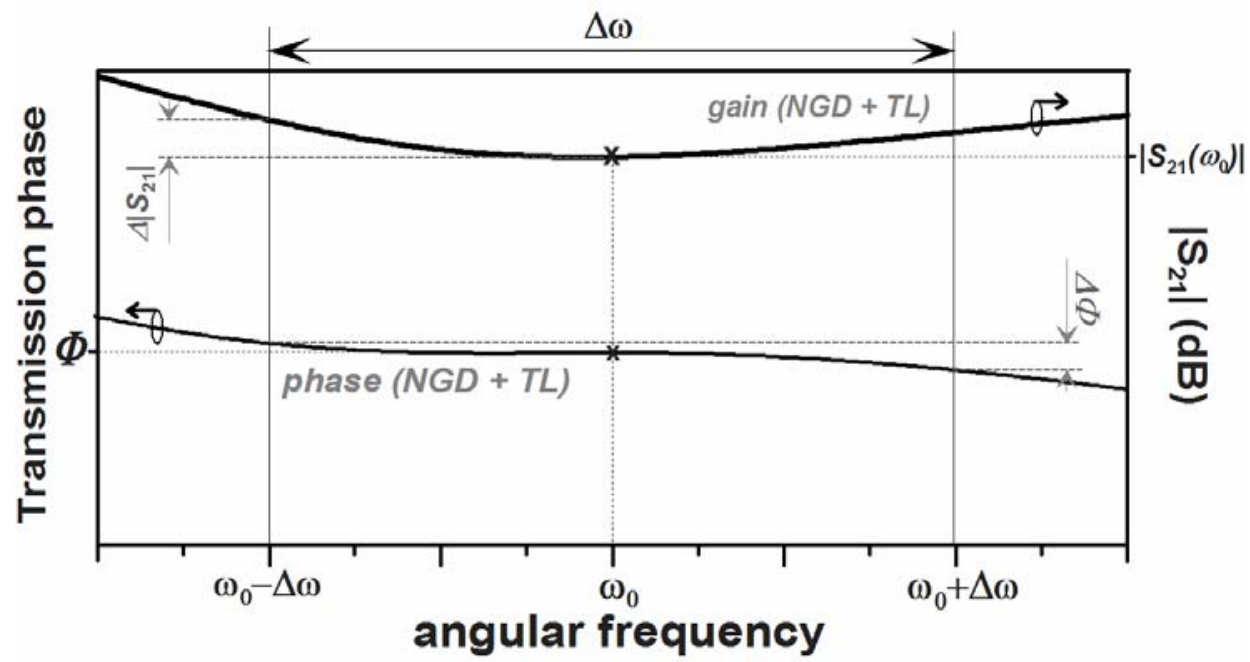

Figure 5. Transmission gain and phase behaviours of an ideal constant NGD PS around a centre angular frequency, $\omega_{0}$.

So, as suggested in [15], using either cascaded NGD cells with different resonant networks or a single cell with several resonant networks in parallel can be a solution to enlarge the NGD bandwidth and thus the constant phase one. This remark was taken into account in the design of a broadband PS prototype reported in the next section.

\section{DESIGN, SIMULATED AND EXPERIMENTAL RESULTS}

This section deals with the design process of a broadband NGD PS through application of the technique developed in section II. It proposes guidelines for the design and implementation procedures before reporting, at first, on the results of simulations carried out with Agilent ADS software, and then on those of the sensitivity assessment achieved through a Monte Carlo analysis. Finally, the results from simulations and measurements are compared and discussed.

\subsection{Design Process}


The FET manufactured by Avago Technology is an ATF-34143. This low noise PHEMT is housed in a 4-lead SC-70 (SOT-343) surface-mount plastic package and can be biased by a single supply voltage. This property allowed us to propose an active bias technique aimed at getting an operating frequency band wider than the one got through classical choke inductor biasing. At first, the FET characteristics required for the synthesis, i.e. $g_{m}=226 \mathrm{mS}$ and $R_{d s}=27 \Omega$, were extracted from the non linear model provided by the manufacturer. Following the synthesis method, the PS component values were calculated in order to get a $145^{\circ}$ phase value and, at least, a $0 \mathrm{~dB}$ gain together with return losses better than $10 \mathrm{~dB}$. In this case of a proof-of-concept circuit, the operating frequency band was kept below $1 \mathrm{GHz}$ to avoid low lumped component values usually accompanied with significant inaccuracies and to minimize the parasitic effects.

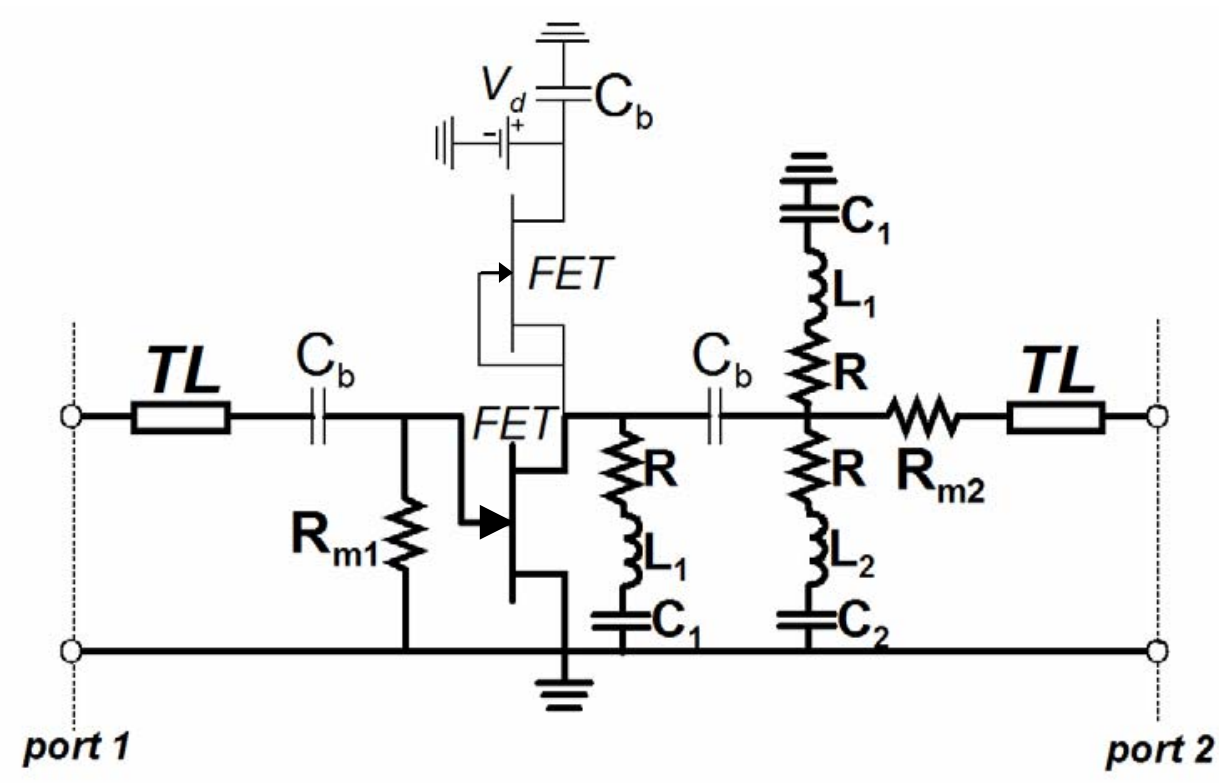

(a) 


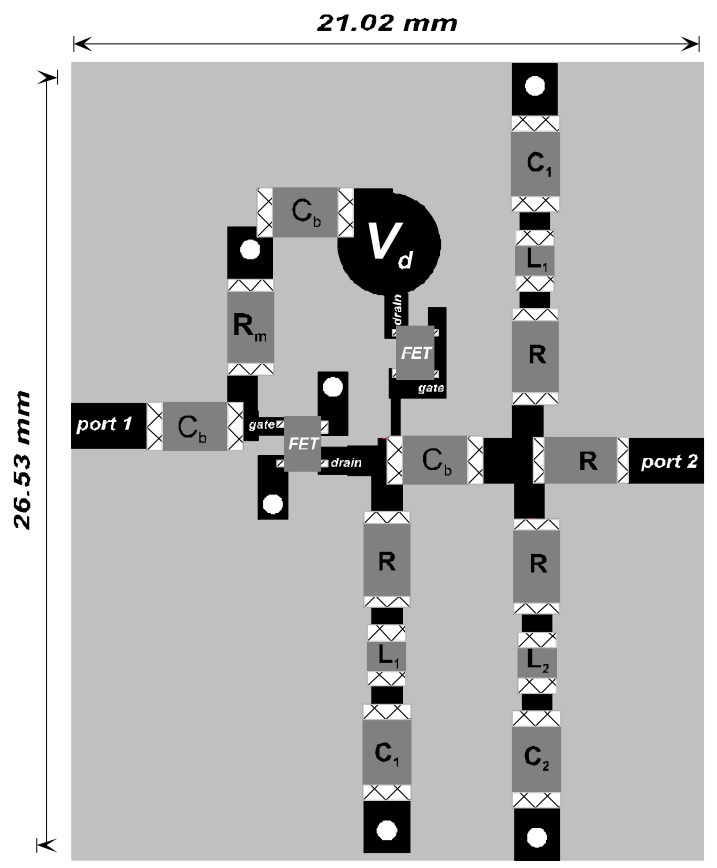

(b)

Figure 6(a). Schematic (bias network in thin lines) and (b) layout of the fabricated phase shifter including the bias network: ATF-34143 PHEMT FET, $V_{d}=2 \mathrm{~V}, V_{g s}=0, I_{d}=100 \mathrm{~mA}$ (capacitors and resistors in surface mount 1206 packages; and inductors in SMT0805) $R_{m}=68 \Omega, R=22 \Omega, L_{1}=5.6 \mathrm{nH}, L_{2}=8 \mathrm{nH}, C_{1}=30 \mathrm{pF}, C_{2}=6.8 \mathrm{pF}, C_{b}=100 \mathrm{nF}$, and, $T L$ $(2 \times d=6.6 \mathrm{~mm})$.

To reach the specified objective of a $150 \%$ relative bandwidth $\left(145^{\circ} \pm 10^{\circ}\right)$ with the components at our disposal, the synthesized RLC network was replaced with a configuration with three-shunt RLC networks. Indeed, in [14], for a single RLC network, the relative bandwidth $\left(90^{\circ} \pm 10^{\circ}\right)$ was restricted to about $85 \%$. It is worth noting that the final values were obtained through a slight optimization performed with the non-linear model of FET and took into account the parasitic effects generated by the layout connecting lines. Indeed, the NFET manufacturer provides an ADS high-frequency non-linear model where the die is modelled by a Statz MESFET model optimized from 0.1 to $6 \mathrm{GHz}$. Figure 6(a) depicts the schematic of the fabricated proof-of-concept circuit and gives the component values, the bias characteristics and the transmission line lengths, whereas Figure 6(b) presents the 
corresponding layout implemented in microstrip hybrid technology and printed on an FR4 substrate (permittivity, $\varepsilon_{r}=4.3$ and thickness, $h=800 \mu \mathrm{m}$ ) is presented in Figures $6(\mathrm{~b})$.

\subsection{Simulations and Monte Carlo Sensitivity Analysis}

At first, the simulations were run on ADS software under the most realistic conditions and use of the most relevant models to check whether the objectives were met. Then, a Monte Carlo analysis was performed in order to assess the influence of the component value tolerances and the impact by eventual design imperfection on the circuit responses.

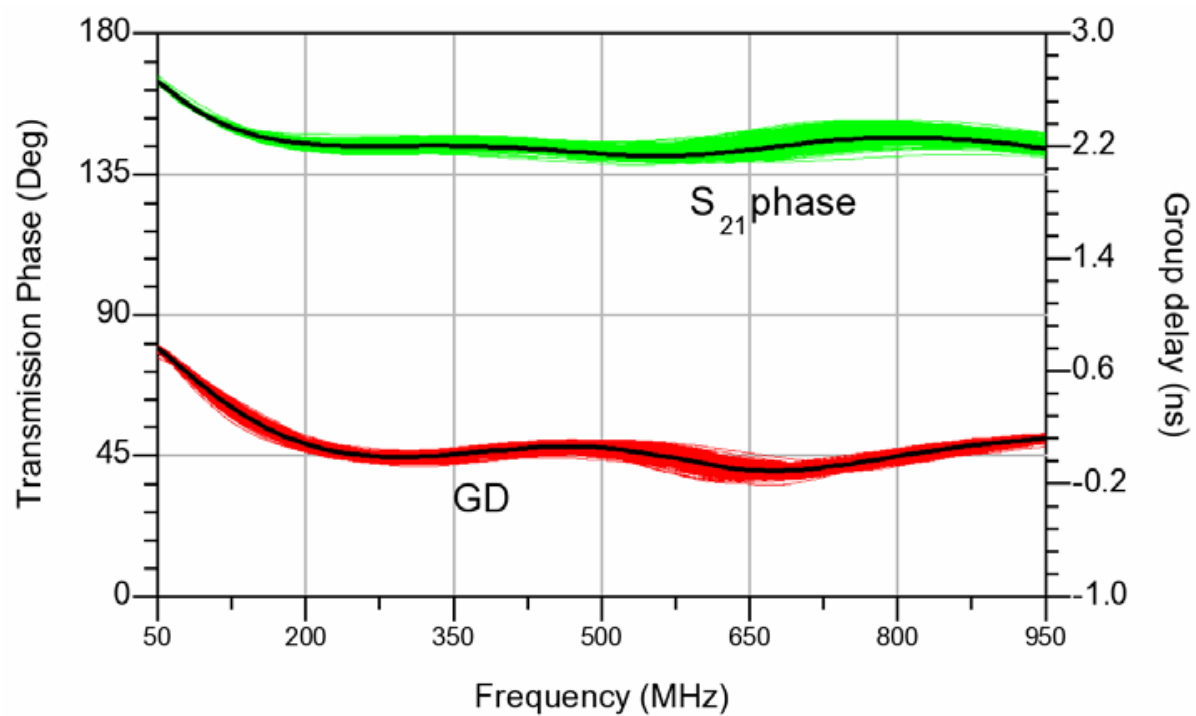

(a)

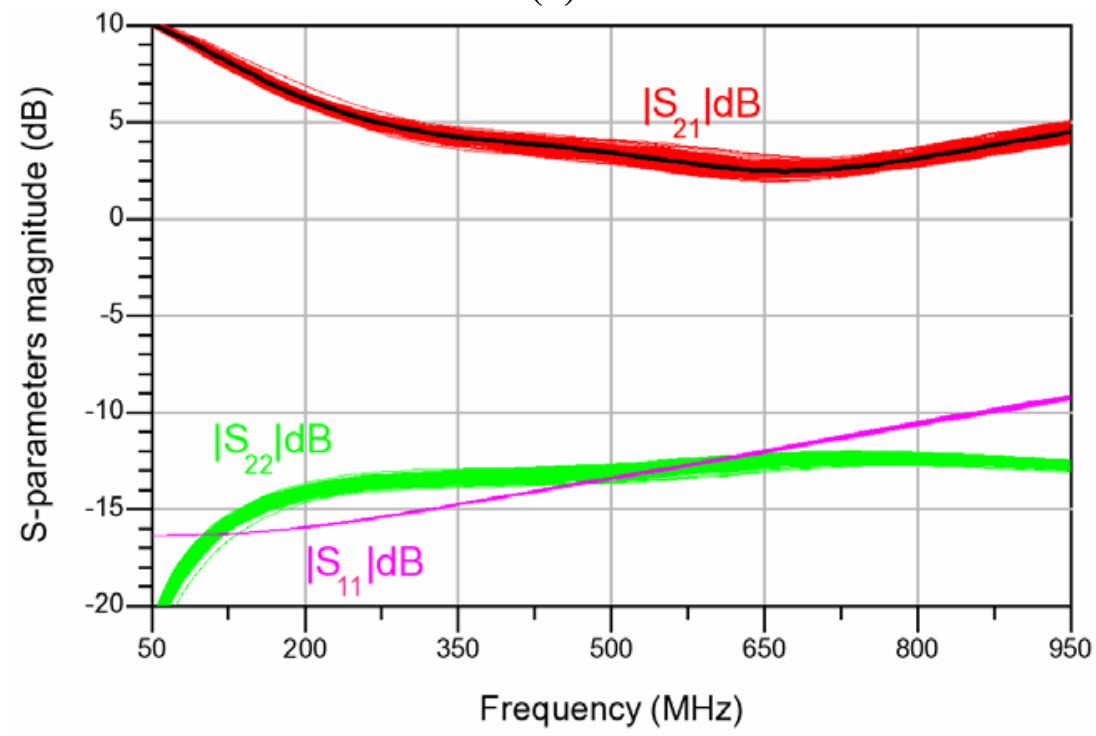

(b) 
Figure 7. Simulation results with a Monte Carlo analysis (300 trials and a 5\%-sensitivity): (a) transmission phase and group delay and (b) S-parameters magnitude.

This analysis was made on 300 trials essentially to gain more insight into the sensitivity: briefly, the values issued from the design for $R, L, C$ (including matching lumped components) and $d$ (TL length) were slightly varied according to a Gaussian statistical distribution.

The simulation results presented in Figure 7 show that the proposed specifications are generally achieved. Indeed, Figure 7(a) demonstrates that the transmission phase nominal curve (black thick curves) is close to $145 \pm 10^{\circ}$ between 50 and $950 \mathrm{MHz}$. The sensitivity is about $13^{\circ}$ against the $\pm 5 \%$ tolerances while remaining in the $145 \pm 10^{\circ}$ range. Figure $7(\mathrm{~b})$ highlights that the magnitudes of S-parameters, $S_{11}, S_{22}$ and $S_{21}$, are close to the specifications: i.e., less than $-10 \mathrm{~dB}$ for $S_{11}$ and $S_{22}$ and between 2.5 and $10 \mathrm{~dB}$ for $S_{21}$. The deviations are less than $1 \mathrm{~dB}$ around the nominal curves. Thus, on the whole, the PS sensitivity versus the component- and line length- tolerances can be considered as not significant. Furthermore, an analysis over a wide bandwidth of the reflection coefficients at the FET accesses in the whole circuit simulation showed us that it was stable. Indeed, $R_{m 1}$ and $R_{m 2}$ were optimized to guarantee the amplifier stability while keeping input-and output-return losses as well as gain at satisfying values.

\subsection{Measurements}

The S-parameters were measured with an E8364A Agilent VNA over the band from $50 \mathrm{MHz}$ up to $10 \mathrm{GHz}$. The results of measurements and simulations are compared in Figure 8. One should note that the stability expected from simulations is confirmed by measurements. In the 50-950 MHz frequency band, the results of measurements about the S-parameters, group 
delay and transmission phase fit those from simulations, especially the group delay and $\mathrm{S}_{21^{-}}$ magnitude values. The phase is $145^{\circ} \pm 10^{\circ}$ in the $102-935 \mathrm{MHz}$ band, which corresponds to a $160 \%$ relative bandwidth $\left(\Delta f / f_{0)}\right.$ with a centre frequency, $f_{0}$, at $528.5 \mathrm{MHz}$. It is worth noting that, on the whole, the difference between simulations and measurements lies within the area defined from the various tolerances.
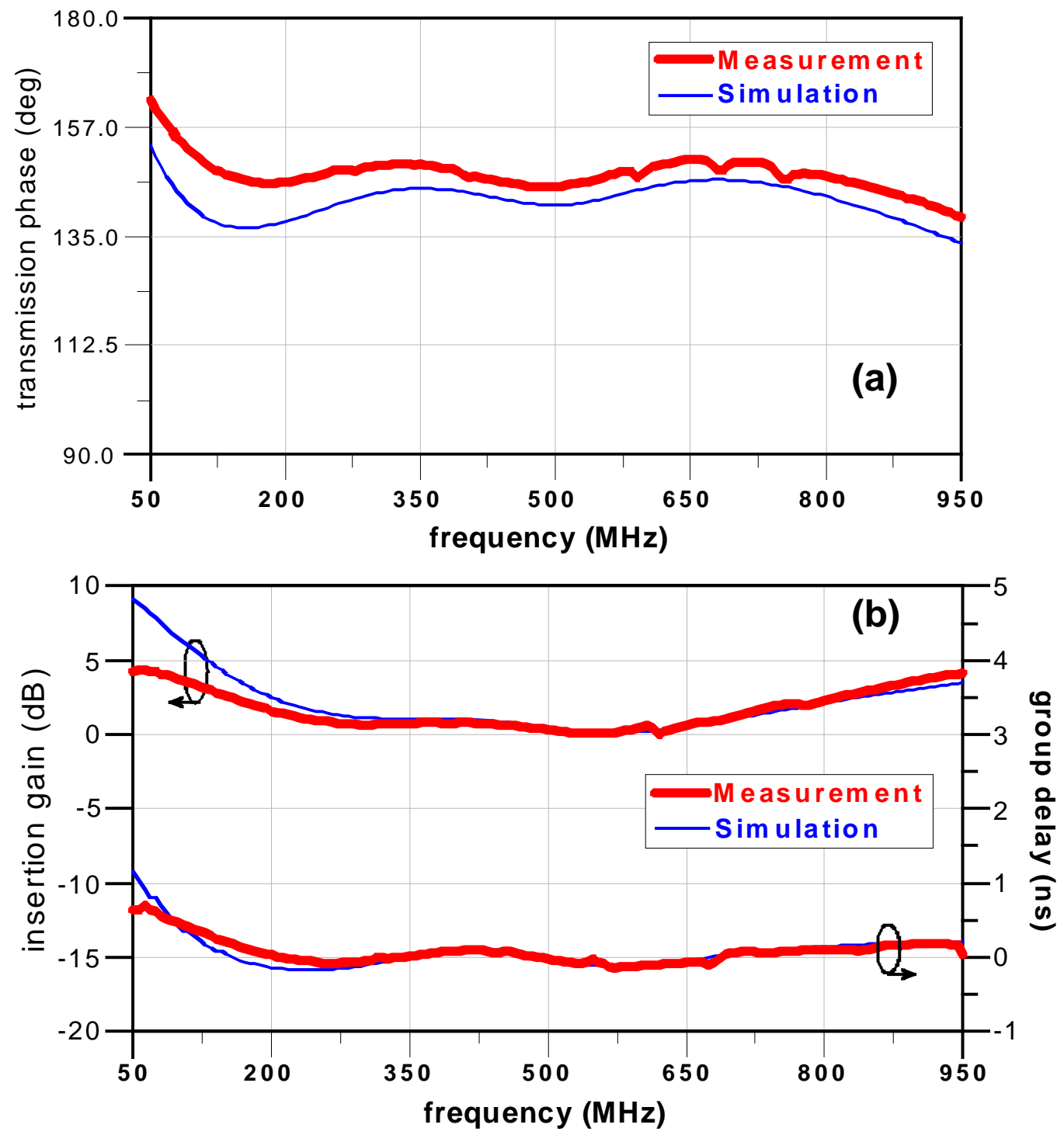


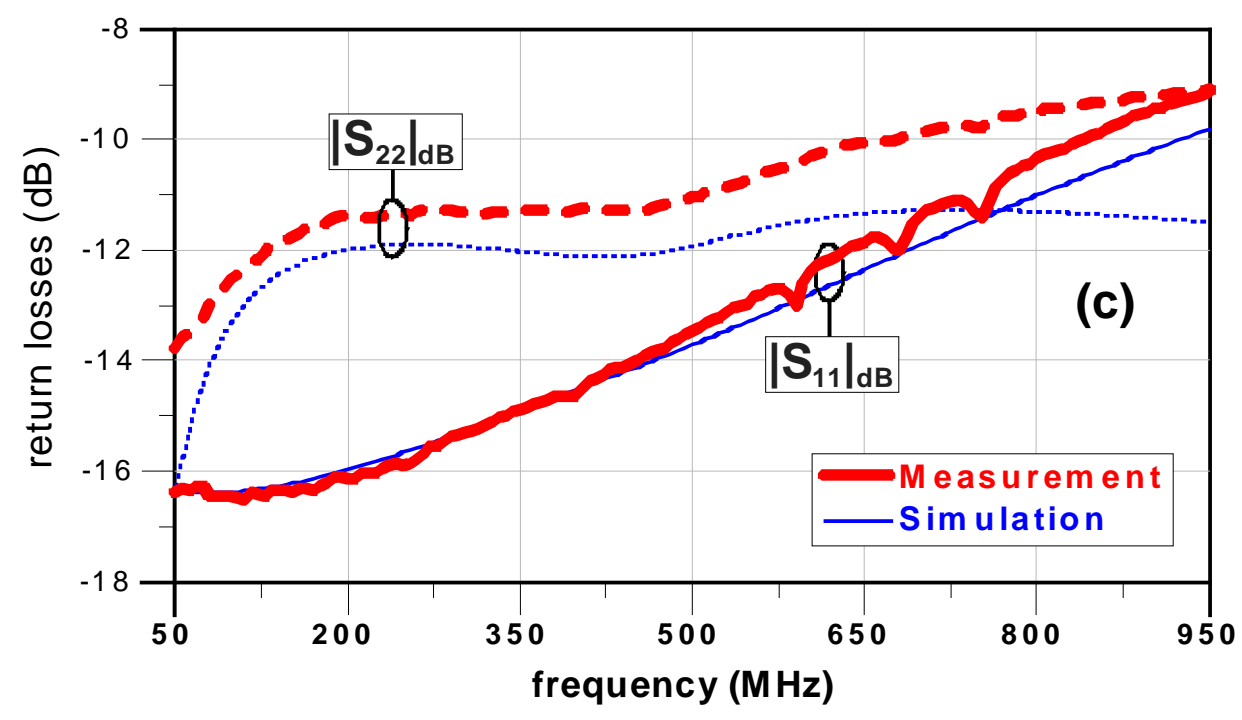

Figure 8. Comparison between simulation and measurement results: (a) Transmission phase frequency responses; (b) Insertion gain and group delay; (c) return losses.

Furthermore, the measurements show an enhancement of the measured gain flatness with respect to the simulation results: indeed, on average the gain is about $2 \mathrm{~dB}$ with variation of $\pm 2 \mathrm{~dB}$ from 50 to $950 \mathrm{MHz}$ (Figure $8(\mathrm{~b})$ ). In this frequency band, the return losses are better than $9 \mathrm{~dB}$ (Figure 8(c)) and a good isolation, at least better than $-22 \mathrm{~dB}$, is realized. The isolation presented by this type of PS may constitute an advantage when an implementation in multi-arm devices such as baluns [18] is envisaged. It is worth underlining that an implementation of constant phase values from $-180^{\circ}$ to $0^{\circ}$ with an even number of NGD cells leads systematically to a smaller line length than the one obtained with an odd number. It is the opposite with the other phase range values.

Non-linear performances were also estimated from simulations by considering the largesignal FET model provided by the manufacturer. Under ADS large-signal harmonic-balance simulations, when biased at $\mathrm{V}_{\mathrm{ds}}=1 \mathrm{~V}$ (i.e. $\mathrm{V}_{\mathrm{d}}=2 \mathrm{~V}$ ) with $\mathrm{I}_{\mathrm{dss}}=132 \mathrm{~mA}$, the active PS provided output power at the $1-\mathrm{dB}$ gain compression point $(\mathrm{P}-1 \mathrm{~dB})$ of $0.5 \mathrm{dBm}$ 
(corresponding to approximately $0-\mathrm{dB}$ gain) at the $500 \mathrm{MHz}$ central frequency. At this frequency, and for a $0-\mathrm{dBm}$ input power, the output harmonics were below the fundamental from $25 \mathrm{~dB}$ which resulted to an acceptable distortion. The device featured 4.9-dB NF (Noise Figure) at $500 \mathrm{MHz}$. Simulations with and without the three resonators showed a contribution of $1 \mathrm{~dB}$ on NF. Large-Signal S-parameters simulations also proved that neither the gain, nor the phase value, nor the flatness were significantly modified up to the $0 \mathrm{dBm}$ as input power value; indeed for this level at $500 \mathrm{MHz}$, the $\mathrm{S}_{21}$ large-signal parameter gap is equal to $1 \mathrm{~dB}$ compared to the small-signal one and the phase value is only modified of less than $1^{\circ}$. These non-linear characteristics validate the use of this PS for rather large signals. Moreover, they illustrate that this NGD PS topology is mostly suited to receiver front-end. Setting the RLC series networks in front of the active components may be a solution to design a PS such that it can precede the radiating element in the emitter front-end in order to deliver larger output signals. Reliability tests about the FET under different temperature conditions are available at the manufacturer web site. Though these measurements like the previous studies showed no significant change in the frequency response versus time or temperature, it may be relevant to plan a complete experimental study of the PS behavior versus temperature.

\section{Conclusion}

A novel frequency-independent phase shifter was successfully synthesized and tested. Compared to the existing PSs in [26-28], its novelty stands in the use of an NGD active circuit that allows one to get constant phase over a broadband. The basic principle relies on the association in cascade of two devices with the same phase slopes in absolute value, but of opposite signs: a classical TL is used as positive group delay, or negative phase slope element, and the positive phase slope is provided by an NGD active circuit initially described in [14-15]. In this report, after a recall of the NGD circuit theory, the corresponding synthesis 
relations were established in detail from the S-parameters analysis of the NGD PS structure under study.

After the theoretical analysis, this NGD active PS concept was validated experimentally through the design of a device using PHEMTs and lumped components. This prototype was implemented in hybrid planar technology and included an active broadband bias technique. Experimental results proved to be in good agreement with simulations: indeed, the constant transmission phase of $145^{\circ} \pm 10^{\circ}$ extended over a $160 \%$ relative band centred around $530 \mathrm{MHz}$, with an insertion gain of about $2 \pm 2 \mathrm{~dB}$. In addition to its broadband and frequency-independent transmission phase, the main advantages of this NGD structure are its simple topology and its compactness as well as loss compensation capability. It is worth emphasizing that the proposed PS presents a constant transmission phase, but not a constant phase shift as often described. Moreover, a very low group delay (theoretically null) is guaranteed regardless of the phase value.

To overcome the operating frequency limitations introduced by the lumped components, and particularly the standard SMT inductors, ongoing researches are focused on the replacement of the resonant network with distributed elements before an MMIC integration. In that case, comparative non-linear and noise studies following the different positions available for the resonant networks should be planned to identify the topologies respectively dedicated to receiver or emitter in RF front-end. The design of a reconfigurable NGD PS is also scheduled; indeed, such a device could be particularly attractive for applications in mobile satellite broadcasting systems, e.g. active phased array antenna as introduced in [24-25], or in IQ modulator/demodulator for use in digital communication systems [22], or to ensure particular functions such as frequency convertor, balun [17], PLL. In that way, tunable active inductances [29] are a particularly interesting idea and may also bring solutions to overcome lumped-inductor frequency limitations. Moreover, owing to its ability to provide gain, this NGD PS may also operate as a multi-function device. 


\section{ACKNOWLEDGMENTS}

The authors would like to thank A. Bourrières and S. Girod from IUT of BREST ("Université de Bretagne Occidentale") for the circuit manufacturing and M. P. Friocourt for helpful advice about English writing.

\section{REFERENCES}

1. L. Brillouin and A. Sommerfeld, Wave Propagation and Group Velocity, Academic Press, New York, pp. 113-137, 1960.

2. R. Y. Chiao, Atomic Coherence Effects which Produce Superluminal (but Causal) Propagation of Wavepackets, Quantum Opt. 6, pp. 359-369, 1994.

3. L. J. Wang, A. Kuzmich and A. Dogariu, Gain-Assisted Supraluminique Light Propagation, Nature 406, pp. 277-279, Jun. 2000.

4. A. Dogariu, A. Kuzmich, H. Cao and L. J. Wang, Superluminal Light Pulse Propagation via Rephasing in a Transparent Anomalously Dispersive Medium, Optics Express, Vol. 8, No. 6, pp. 344-350, 12 Mar. 2001.

5. R. Y. Chiao, Faster than Light Propagations, Negative Group Delays, and their Applications, The Physics of Communication Proc. of the XXII Solvay Conference on Physics, DOI No. 10.1142, pp. 287-314, Nov. 2001.

6. D. Solli, R. Y. Chiao and J. M. Hickmann, Superluminal Effects and Negative Delays in Electronics, and their Applications, Phys. Rev. E, Vol. 66, No. 5, pp. 056601.1056601.4, Nov. 2002.

7. M. Kitano , T. Nakanishi and K. Sugiyama, Negative Group Delay and Superluminal Propagation: An Electronic Circuit Approach, IEEE J. Sel. Top. in Quantum Electron., Vol. 9, No. 1, pp. 43-51, 2003. 
8. T. Nakanishi, K. Sugiyama and M. Kitano, Demonstration of Negative Group Delays in a Simple Electronic Circuit, Amer. J. Phys., Vol. 70, Issue 11, pp. 1117-1121, 2002.

9. N. Fang, D. Xi, J. Xu, M. Ambati, W. Strituravanich, C. Sun and X. Zhang, Ultrasonic Metamaterials with Negative Modulus, Nature Materials, pp. 452-456, Jun. 2006.

10. W. M. Robertson, J. Pappafotis, P. Flannigan, J. Cathey, B. Cathey and C. Klaus, Sound Beyond the Speed of Light: Measurement of Negative Group Velocity in an Acoustic Loop Filter, App. Phys. Letters, Vol. 90, No. 1, pp. 014102.1-014102.3, 2007.

11. G. V. Eleftheriades, A. K. Iyer and P. C. Kremer, Planar Negative Refractive Index Media Using Periodically L-C Loaded Transmission Lines, IEEE Trans. MTT, Vol. 50, Issue 12, pp. 2702-2712, Dec. 2002.

12. O. F. Siddiqui, M. Mojahedi and G. V. Eleftheriades, Periodically Loaded Transmission Line With Effective Negative Refractive Index and Negative Group Velocity, IEEE Trans. Antennas Propagat., Vol. 51, No. 10, pp. 2619-2625, Oct. 2003.

13. O. F. Siddiqui, S. J. Erickson, G. V. Eleftheriades and M. Mojahedi, Time-Domain Measurement of Negative Group Delay in Negative-Refractive-Index TransmissionLine Metamaterials, IEEE Trans. MTT, Vol. 52, No. 5, pp. 1449-1454, May 2004.

14. B. Ravelo, A. Pérennec, M. Le Roy and Y. Boucher, Active Microwave Circuit with Negative Group Delay, IEEE MWC Lett., Vol. 17, Issue 12, pp. 861-863, Dec. 2007.

15. B. Ravelo, A. Pérennec and M. Le Roy, Synthesis of Broadband Negative Group Delay Active Circuits, IEEE MTT-S Symp., Honolulu (Hawaii), pp. 2177-2180, 2-9 June 2007.

16. B. Ravelo, M. Le Roy and A. Perennec, Application of Negative Group Delay Active Circuits to the Design of Broadband and Constant Phase Shifters, Microwave Opt. Technol. Letters, Vol. 50, No. 12, pp. 3078-3080, Dec. 2008. 
17. B. Ravelo, A. Pérennec and M. Le Roy, Broadband Balun Using Active Negative Group Delay Circuit, 37th European Microwave Conference, Munich, Germany, pp. 466-469, 8-12 Oct. 2007.

18. R. V. L. Hartley, US. Patent 1666 206, 1928.

19. Z. Kiyasu, On the Wave Separator for Phase Difference, J. Elec. Comm. Engrs., (Japan), Vol. 25-27, pts. I-IV, 1942-1944.

20. S. Darlington, Realization of a Constant Phase Difference, Bell Syst. Tech. J., Vol. 29, pp. 94-104, Jan. 1950.

21. H. R. Phelan, Spiraphase - A New, Low Cost, Lightweight Phased Array, Microwave J., Vol. 19, pp. 41-44, Dec. 1976.

22. H. R. Phelan, Dual Polarization Spiral Antenna, U.S. patent No. 3,906,514, Sep. 16, 1975.

23. J. J. H. Wang, Characteristics of a New Class of Diode-Switched Integrated Antenna Phase Shifter, IEEE Tran. Ant. Prop., Vol. AP-31, pp. 156-159, Jan. 1983.

24. J. J. H. Wang, J. K. Tillery, G. T. Thompson, K. E. Bohannan, R. M. Najafabadi and M. A. Acre, A Multioctave-Band Photonically-Controlled, Low-Profile, StructurallyEmbedded Phased Array with Integrated Frequency Independent Phase-Shifter, IEEE International Symposium on Phased Array Systems and Technology, Boston, MA, pp. 68-73, Oct. 15-18, 1996.

25. Y. J. Ko, J. Y. Park and J. U. Bu, Integrated RF MEMS Phase Shifters with Constant Phase Shift, IEEE MTT-S Symp., Philadelphia, pp. 1489-1492, June 2003.

26. A. M. Abbosh, Ultra-Wideband Phase Shifters, IEEE Trans. MTT, Vol. 55, No. 9, pp. 1935-1941, Sep. 2007.

27. Z. Zhao, X. Wang, K. Choi, C. Lugo aVnd A. T. Hunt, Ferroelectric Phase Shifters at 20 and 30 GHz, IEEE Trans. MTT, Vol. 55, No. 2, pp. 430-437, Feb. 2007. 
28. S. Amari, U. Rosenberg and R. Wu, In-line Pseudoelliptic Band-Reject Filters with Nonresonating Nodes and/or Phase Shifts, IEEE Trans. MTT, Vol. 54, No. 1, pp. 428436, Jan. 2006.

29. U. L. Rohde, A. K. Poddar, Active Inductors Tune Low-Noise VCOs, Microwaves \& RF, ED Online ID \#22570, Apr. 2010.

\section{BIOGRAPHIES}

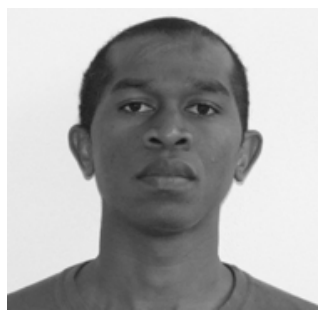

Blaise Ravelo was born in Tananarivo (Madagascar) in 1978. He received his master's and $\mathrm{PhD}$ degrees in electronic \& telecommunication from Brest University, resp. in 2004 and 2008. Currently, he works at IRSEEM (Institute of research on embedded electronic system) laboratory and ESIGELEC engineering school as a Researcher-Lecturer. At the Lab-STICC, he worked on the innovative idea consisting in designing negative group delay active circuits for microwave applications. Currently, he develops EM near-field measurement techniques and treatments, and the EMI modelling of high frequency devices for the EMC applications. E-mail: Blaise.Ravelo@yahoo.fr.

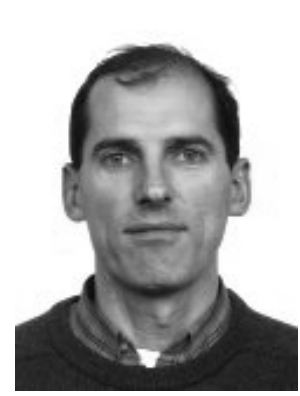

André Pérennec was born in Quimper, France, in 1960. He received the Ph.D degree in electronics from the "Université de Bretagne Occidentale" (France) in 1988. Presently, he is assistant professor in the Lab-STICC (UMR CNRS 3192), Brest, France. His research interests are in the active and passive device microwave area and include amplifier design (low-noise and broad-band), and filters. He also works on optoelectronic and microwave periodic devices. Currently, his main research interests concern circuits with negative group delay. 
Marc Le Roy was born in Brest, France, in 1970. He received the Ph.D. degree in electronics from the LEST (Laboratory for Electronics and Telecommunication Systems, UMR CNRS 6165) at the University of Bretagne Occidentale, Brest, France, in 1999. He re-joined the Lab-STICC (UMR CNRS 3192, formerly LEST) in 2003 as assistant professor. His research activities deal with modelization and design of periodic structures for microwave systems and active devices generating negative group delay. 